Research Paper

\title{
Verification of the Efficacy of New Diagnostic Criteria for Retropharyngeal Nodes in a Cohort of Nasopharyngeal Carcinoma Patients
}

\author{
Dom-Gene Tu${ }^{1}$, Hsuan-Yu Chen², Wei-Jen Yao1, Yu-Sheng Hung 3 , Yu-Kang Chang, ${ }^{4}$ Chih-Han Chang ${ }^{5}$, \\ Yu-Wen Wang6,7凶
}

1. Department of Nuclear Medicine, Ditmanson Medical Foundation Chia-Yi Christian Hospital, Chia-Yi, Taiwan.

2. Institute of Statistical Science, Academia Sinica, Taipei, Taiwan

3. Division of Nuclear Medicine, Department of Medical Imaging, Chi Mei Medical Center, Liouying, Tainan, Taiwan.

4. Department of Medical Imaging Chi Mei Medical Center, Liouying, Tainan, Taiwan.

5. Department of Biomedical Engineering, National Cheng Kung University, Tainan, Taiwan.

6. Department of Radiation Oncology, Ditmanson Medical Foundation Chia-Yi Christian Hospital, Chia-Yi, Taiwan.

7. Department of Radiation Oncology, Chi Mei Medical Center, Liouying, Tainan, Taiwan.

$\square$ Corresponding author: Yu-Wen Wang, MD PhD, E-mail: 02364@cych.org.tw. Department of Radiation Oncology, Ditmanson Medical Foundation Chia-Yi Christian Hospital, 539 Zhongxiao Road, Chia-Yi, 600566, Taiwan.

(1) The author(s). This is an open access article distributed under the terms of the Creative Commons Attribution License (https://creativecommons.org/licenses/by/4.0/). See http:/ /ivyspring.com/terms for full terms and conditions.

Received: 2021.01.19; Accepted: 2021.08.02; Published: 2021.08.13

\begin{abstract}
Purpose: A multistage approach to diagnose lateral retropharyngeal nodes (LRPNs) of nasopharyngeal carcinoma (NPC) had been proposed and warranted for validation.

Methods: Between 2012 and 2017, the patients with newly diagnosed NPC were enrolled. The responsive nodes or those that progressed during follow-up were positive. The criteria for the multistage approach delimited LRPNs with a minimal axial diameter (MIAD) $\geq 6.1 \mathrm{~mm}$ were assessed as positive and if the mean standard uptake value $\geq 2.6$, or if the maximal coronal diameter $\geq 25 \mathrm{~mm}$ and maximal axial diameter $\geq 8 \mathrm{~mm}$ with nodes MIAD $<6.1 \mathrm{~mm}$ were also considered as positive. The outcomes were compared with the MIAD cutoff value $\geq 6 \mathrm{~mm}$ (traditional method). A chi-squared test was used to compare two areas under the curve of the receiver operating characteristic curves.
\end{abstract}

Results: A total of 67 eligible NPC cases and 155 LRPNs (72 positive and 83 negative) were analyzed. The accuracy, specificity, and sensitivity of the traditional method were $0.91,0.93$, and 0.89 , respectively. The values for the multistage approach all reached 0.94 . The area under the curve was significantly greater for the multistage approach compared with the traditional method $(p=0.023)$.

Conclusion: The results support the advantage of the multistage approach.

Key words: diagnosis, nasopharyngeal carcinoma, retropharyngeal nodes, radiotherapy

\section{Introduction}

The accurate diagnosis of lateral retropharyngeal nodes (LRPNs) with images for nasopharyngeal carcinoma (NPC) is important. Magnetic resonance imaging (MRI) is considered as a golden standard for detecting LRPNs in NPC [1]. Based on control subject analysis, Lam and King used minimal axial diameter (MIAD) of 4 and $5 \mathrm{~mm}$ as the upper limit of normal LRPNs [2,3]. A diameter of $5 \mathrm{~mm}$ or higher as a criterion of malignancy was widely indicated by previous studies [4-12]. However, Zhang et al. reported that $\geq 6 \mathrm{~mm}$ diameter might be a better cutoff point for malignancy [13]. The method proposed by Zhang was reviewed as a "robust standard methodology" [14]. A better clinical prediction was also obtained by Li et al. using a MIAD of $6 \mathrm{~mm}$ [15]. The newly proposed size criterion for malignant LRPNs minimally up-shifted the definition of LRPN involvement from " $\geq 5 \mathrm{~mm}^{\prime}$ to "> $5 \mathrm{~mm}$," with the consensus of an international guideline for NPC and several publications [16-20]. However, based on the prognostic value of LRPN metastasis laterality, a recent study [21] favored $\geq 5 \mathrm{~mm}$ as more suitable than 
$\geq 6 \mathrm{~mm}$ as the cutoff value of MIAD. The inconsistency of results in the above-published data characterizing LRPNs implies that when only a single factor is used to determine the malignancy of LRPNs, the power of detection could be limited. 2-[ $\left.{ }^{18} \mathrm{~F}\right]-$ F-fluorodeoxyglucose (FDG) positron emission tomography (PET)/computed tomography (CT), with its functional imaging sensitivity in detecting cancerous nodal lesions, may be complementary to MRI, which was highly encouraged in the 2017 American Joint Committee on Cancer staging system [22]. Previously, we proposed a diagnostic criteria of parameters combined mean standard uptake value (SUV) from FDG PET/CT and MRI (MIAD, maximal axial diameter, and maximal coronal diameter) with a significantly higher reported accuracy. This criterion is still subject to external review despite its higher reported accuracy [23]. Using a multistage approach to assess a node in daily practice can be cumbersome. Thus, the superiority of the new criteria must be verified to convince clinicians to adopt them. This study aimed to validate the efficacy of the novel multistage approach to diagnose LRPNs with a new cohort of NPC cases.

\section{Methods}

This study was approved by the Institutional Review Board of the Chi Mei Medical Center (Approval numbers: 10710-L06). Although consent was not specifically obtained for this retrospective review, all information was anonymized and de-identified before its analysis.

\section{Patients and treatment}

Between Oct 2012 and Dec 2017, the patients with newly diagnosed NPC were enrolled. Exclusion criteria included cases without MRI or FDG PET, examining dates within 3 weeks and before starting any cancer treatment, cases with incomplete definitive RT dose (under 59.4 Gy), those who did not undergo MRI within 3 months after the end of RT date, those whose initial MRI failed to reveal any LRPN, and those lacking tissue proof of NPC. Patients with additional head and neck cancers or acute inflammation were also excluded from this study. All patients received RT-based cancer treatment (including induction chemotherapy + concurrent chemoradiation, chemoradiation \pm adjuvant chemotherapy, or RT alone). RT was given with standard fractionations with dose ranging from 59.4 Gy to $72 \mathrm{~Gy}$, with mean \pm standard deviation (SD) = $70.73 \pm 1.66$ Gy. All the patients received RT with intensity-modulated RT or volumetric arc therapy with an accelerator or TomoTherapy.

\section{Imaging protocol and assessment}

All the patients underwent initial MRI and PET/CT. MRI and FDG PET/CT scans were conducted less than 3 weeks apart (mean $\pm \mathrm{SD}=2.5 \pm$ 6.0; range: 0-17 days) before cancer treatment. The details of imaging protocols for MRI (Siemens Medical Systems, Erlangen, Germany) and PET/CT (GE Healthcare, Chicago, IL, USA) and the methods of measurement of nodal parameters were identical to those described in our previous reports [23, 24]. Three experienced NPC physicians blinded to patients' details evaluated for this study. A radiation oncologist and a nuclear medicine physician evaluated both MRI images and the corresponding FDG PET/CT data and the mean SUV of the FDG PET/CT data for the region of interest using the MRI image as an anatomical reference. Any disagreements were resolved by consensus among the three physicians.

\section{Follow-up and Assessment of Lymph Nodes}

The images of LRPNs were reviewed. We observed the response of these nodes before and after RT-based local treatment by serial MRI. Repeated MRI was performed within 1-2 months (mean \pm SD = $43.9 \pm 15.7 ; 6-85$ days) after RT. Positive nodes could be identified for patients with a follow-up of fewer than 6 months. The absence of nodal recurrence of more than 6 months was needed if negative nodes were diagnosed. Overall, the follow-up period after RT ranged from 0.2 months to 84.4 months (mean \pm $\mathrm{SD}=30.1 \pm 20.9$ ). We measured the changes in maximal axial diameter and maximal coronal diameter before and after the treatment to determine the nature of nodes $[23,25]$. The responsive nodes and those that progressed during follow-up were positive; otherwise, the nodes were considered negative (Fig.1). The images of widely accepted characteristics of an involved node, as recommended by international consensus of delineation of target volume for NPC extracapsular extension, central necrosis, and three or more contiguous confluent LRPNs in MRI and overt FDG avid node in PET/CT, were also recorded in addition to the nodal diameter for comparison [16].

Currently, we think that the optimal conventional method with a single parameter criterion (MIAD $\geq 6.0 \mathrm{~mm}$ ) yielded positive results, although we also tested the outcome results by using MIAD $\geq 5.0 \mathrm{~mm}$ for comparison [13-15, 23]. The proposed new criteria with the multistage approach included MIAD and mean SUV from the FDG-PET and MRI (maximal axial diameter and maximal coronal diameter) could be predictors. The LRPNs with a MIAD $\geq 6.1 \mathrm{~mm}$ were considered positive. If the mean $\mathrm{SUV} \geq 2.6$ or if the maximal coronal diameter $\geq 25 \mathrm{~mm}$ and maximal axial diameter $\geq 8$ 
$\mathrm{mm}$, the nodes with MIAD $<6.1 \mathrm{~mm}$ should be considered as positive [23]. Otherwise, they were negative (Fig. 2).

\section{Statistical Analysis}

The scatter plots for each parameter for LRPNs for positive and negative nodes were illustrated with Excel 2010 version 14.0.7212.5000 (Microsoft, Redmond, WA). The new approach and MIAD $\geq 6.0$ $\mathrm{mm}$ were tested to derive the respective accuracy for LRPNs, which were indicated by the receiver operating characteristic curve. For comparison of the difference between the two methods, the significance of the difference between the two areas under the curves (AUC) from both approaches was calculated by the Chi-squared test with the null hypothesis considering the two areas under the curves as equal. The statistical analyses were carried out using SAS 9.4 (Cary, NC, USA).

\section{Results}

A total of 137 patients were initially enrolled for the investigation. Table 1 lists the 67 eligible NPC cases and their clinical characteristics. Among the 155 LRPNs identified from these patients, 72 were positive, and 83 were negative (Fig. 1, with an additional table showing details [see Supplementary Table S1]). All positive and negative nodes for MIAD, maximal axial diameter, maximal coronal diameter, and mean SUV were separately drawn with scatter plots (Fig. 3, with additional tables showing details [see Supplementary Table S1 and S2 respectively]). The results were assessed by using three main qualifications: accuracy: number of correct assessments over the number of all assessments, meant to measure the degree of veracity of a diagnostic test on a condition; specificity: number of true negative assessment over the number of all negative assessment, suggesting how good the test is at identifying negative condition; and sensitivity: number of true positive assessment over the number of all positive assessment, suggesting how good the test is at detecting a disease. The results from traditional MIAD cutoffs at $\geq 5 \mathrm{~mm}$ reached $0.85,0.74$, and 0.97 , respectively. The values at $\geq 6 \mathrm{~mm}$ were 0.91 (accuracy), 0.93 (specificity), and 0.89 (sensitivity). The accuracy, specificity, and sensitivity for the multistage approach were all 0.94 (Table 2). Compared with that of the traditional method, the area under the curves (Fig. 4) was significantly greater for the multistage method with a cutoff at $\geq 6 \mathrm{~mm}$ with $\mathrm{p}=0.023$. In our series, 28 LRPNs with a picture of node three or more contiguous confluent, 39 extracapsular extensions, 44 central necrosis, and 40 overt FDG uptake LRPNs were obtained and identified as positive nodes. There is an additional table showing details [see Supplementary Table S3].

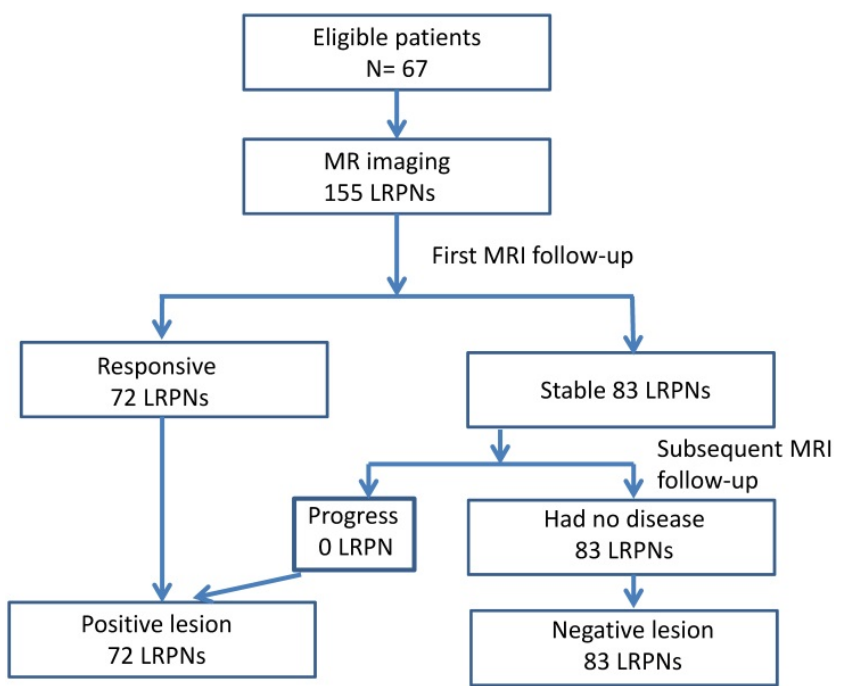

Figure 1. Flowchart outlining the follow-up MRI results of 155 lateral retropharyngeal nodes in 67 patients.

Table 1. Clinical characteristics of 67 eligible nasopharyngeal carcinoma patients

\begin{tabular}{lc}
\hline Characteristic & Number of patients (percentage) \\
\hline Age (years) & $15(22)$ \\
Age $<40$ & $52(78)$ \\
Age $\geq 40$ & \\
Sex & $46(69)$ \\
Male & $21(31)$ \\
Female & $0(0)$ \\
World Health Organization pathologic feature \\
Keratinizing squamous cell carcinoma & $9(13)$ \\
Non-keratinizing carcinoma & \\
(not otherwise specified) & \\
Non-keratinizing differentiated carcinoma & $23(34)$ \\
Non-keratinizing undifferentiated & $34(51)$ \\
carcinoma & $1(1)$ \\
Not applicable & \\
American Joint Committee on Cancer 2010 stage \\
I & $3(4)$ \\
II & $11(16)$ \\
III & $23(34)$ \\
IV & $30(45)$
\end{tabular}

Table 2. Accuracy results of the multistage and conventional criteria for 155 lateral retropharyngeal nodes in our patients

\begin{tabular}{|c|c|c|c|c|c|}
\hline Criteria for 155 nodes & Accuracy & Specificity & Sensitivity & Positive predictive value & Negative predictive value \\
\hline MIAD $\geq 5.0 \mathrm{~mm}$ & 0.845 & 0.735 & 0.972 & 0.761 & 0.968 \\
\hline MIAD $\geq 6.0 \mathrm{~mm}$ & 0.910 & 0.928 & 0.889 & 0.914 & 0.906 \\
\hline Multi-stage method & 0.942 & 0.940 & 0.944 & 0.932 & 0.951 \\
\hline
\end{tabular}

MIAD: minimal axial diameter. 


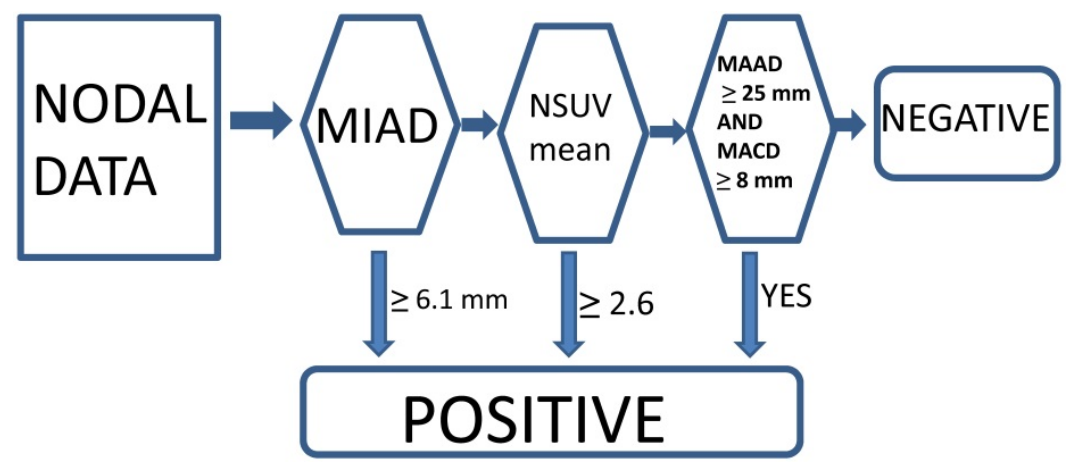

Figure 2. Multistage approach with new criteria for lateral retropharyngeal nodes in retropharyngeal carcinoma.
A

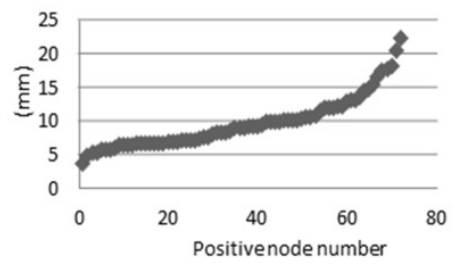

B

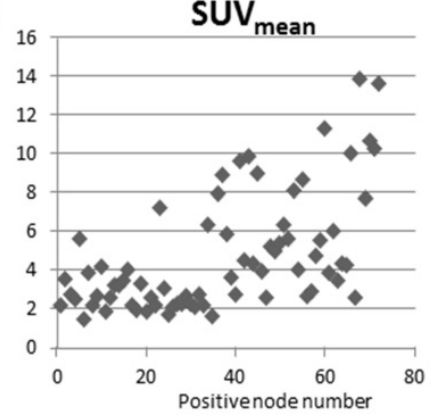

C

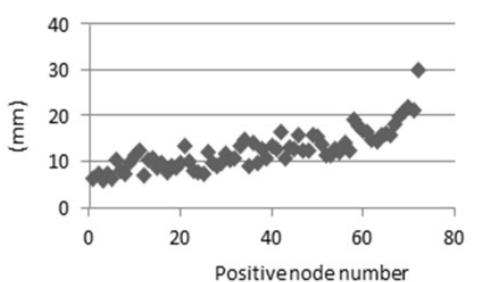

D

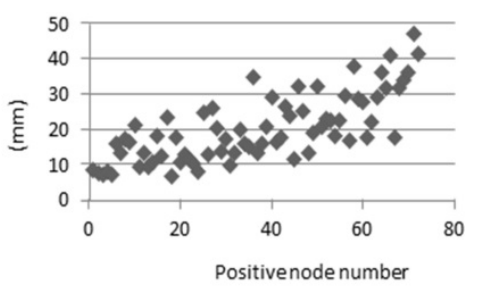

MIAD

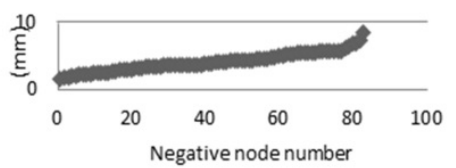

SUV $_{\text {mean }}$

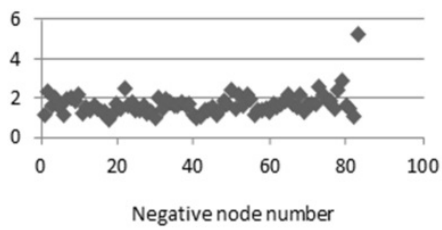

MAAD

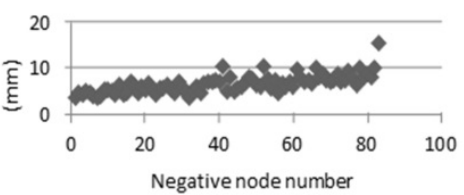

MACD

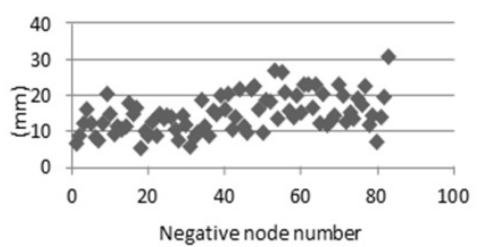

Figure 3. Scatter plots of parameters versus 72 positive nodes on the left side and 83 negative nodes on the right side. A: We plotted the minimal axial diameter (MIAD) with the node numbers in abscissa ranked by the MIAD size. The values of other parameters (B: mean standard uptake value (SUV ${ }_{\text {mean) }}$; $\mathbf{C}$ : maximal axial diameter (MAAD); D: maximal coronal diameter (MACD)) are correspondingly plotted.

\section{Discussion}

Most authors agreed the criterion of LRPNs selected the MIAD ranging from $\geq 5$ to $\geq 6 \mathrm{~mm}$ in the past two more decades [3-21]. As a single parameter, MIAD was proved to be more useful than the maximal axial diameter by Zhang's study [13]. In that article, they used the responsiveness and follow-up data to judge LRPNs. This method was reviewed to be robust [14]. However, shifting MIAD $\geq 6 \mathrm{~mm}$ from $\geq 5 \mathrm{~mm}$ was not accepted worldwide for clinical use due to the survival data and its inconsistency [15, 21]. Our findings were consistent with the results showing a higher accuracy to be from MIAD $\geq 6 \mathrm{~mm}$, not from MIAD $\geq 5 \mathrm{~mm}$ (Tables 1 and 2). Therefore we support the single cutoff value of MIAD should be up-shifted to $6 \mathrm{~mm}$.

Specificity and sensitivity can never be enhanced simultaneously by tuning a single parameter, problems will remain unsettled if we do not consider adding other parameters for assessment. Table 2 showed better diagnostic outcomes compared with those from either MIAD $\geq 6 \mathrm{~mm}$ or $\geq 5$ $\mathrm{mm}$. The performance improvement of this multistage approach reinforced the benefit of applying our method compared to previous ones [23]. Compared with our previous study (Table 3), the current findings were better in all outcome categories. A possible explanation was that the tested nodes might be easier to be judged in this cohort. However, the advantage of both cohorts for the multistage method over a single parameter was the enhancement of specificity and sensitivity, different from shifting a single cutoff value from $5 \mathrm{~mm}$ to $6 \mathrm{~mm}$ that results in the sacrifice of sensitivity. Under the framework of multistage approach, those nodes with a MIAD smaller than $6.1 \mathrm{~mm}$ (after step 1 in Fig. $2)$, additional inclusion of factors such as nodal mean SUV $\geq 2.6$, or maximal axial and coronal diameter $\geq 8 \mathrm{~mm}$ and $\geq 25 \mathrm{~mm}$ become relevant points to identify false-negatives, as had been mischaracterized by previous methodologies. 
Table 3. Results of multistage and two conventional criteria for 410 nodes in our previous report [23]

\begin{tabular}{|c|c|c|c|c|c|}
\hline Criteria for 410 nodes & Accuracy & Specificity & Sensitivity & Positive predictive value & Negative predictive value \\
\hline MIAD $\geq 5.0 \mathrm{~mm}$ & 0.846 & 0.779 & 0.910 & 0.814 & 0.891 \\
\hline MIAD $\geq 6.0 \mathrm{~mm}$ & 0.890 & 0.930 & 0.853 & 0.928 & 0.856 \\
\hline Multi-stage method & 0.905 & 0.950 & 0.863 & 0.948 & 0.867 \\
\hline
\end{tabular}

MIAD: minimal axial diameter.

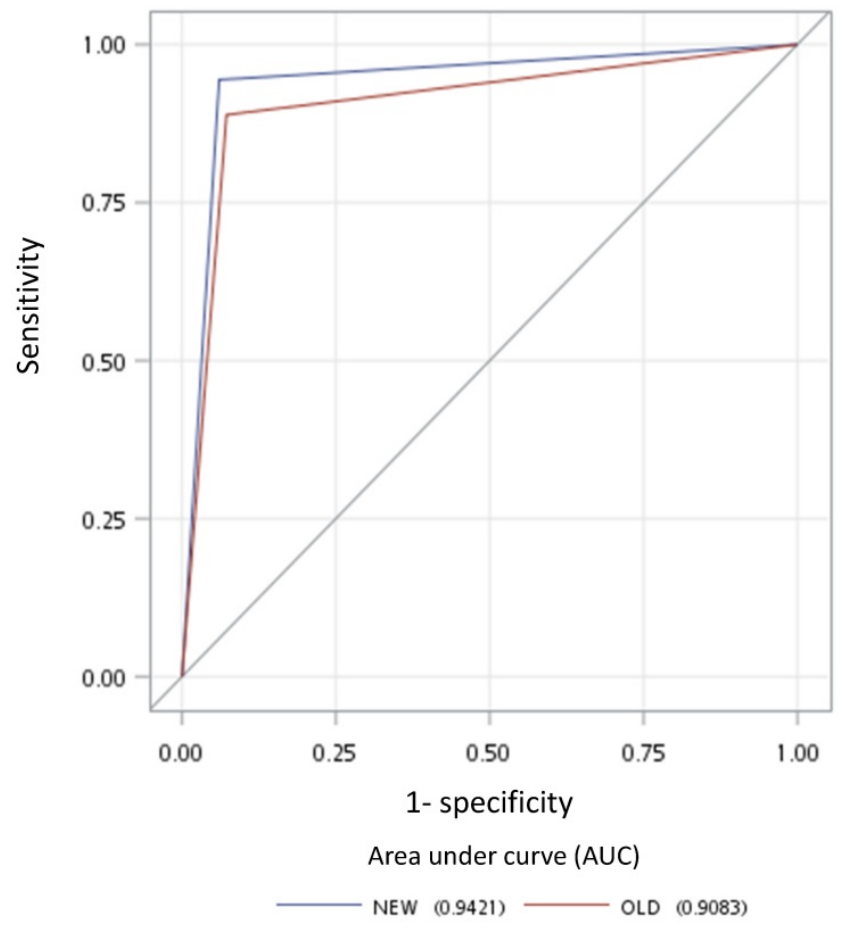

Figure 4. Receiver operating characteristic curves for the new multistage approach (blue line) and old criterion using minimal axial diameter cutoff at $\geq 6 \mathrm{~mm}$ (red line).

Table 4. Predicted conditions of the multistage and conventional criteria for 155 lateral retropharyngeal nodes in our patients

\begin{tabular}{llll}
\hline & \multicolumn{2}{c}{ MIAD $\geq 6.0 \mathrm{~mm}$} & Total \\
\cline { 2 - 3 } & Negative & Positive & \\
\hline Multi-stage method & 81 & 1 & 82 \\
Negative & 4 & 69 & 73 \\
Positive & 85 & 70 & 155 \\
Total & & & \\
\hline
\end{tabular}

MIAD: minimal axial diameter.

PET/CT has been increasingly used in NPC patients because of its sensitivity in detecting distant metastasis (M1) and secondary cancer [22]. However, the role of FDG-PET/CT alone in determining the involvement of LRPNs is rarely appreciated [24, 26]. As a component of the multistage method, we find the overall accuracy of diagnosis can still be enhanced remarkably by the evaluation of mean SVU. Since LRPN is a special part of the NPC nodal region, our special type of diagnostic criteria demonstrates practical guidance on how the supplementing role of PET/CT can be played, as suggested in the $8^{\text {th }}$ edition of the AJCC staging system. This finding is suggested to be included in the supporting data for NPC nodal staging system in the next version.

Overall in our 155 LRPNs, there were five nodes with different outcomes judged from two methods (Table 4). All nodes with different results showed the new method to be correct. The improved performance of the new method over the traditional method was proved to be statistically different in the AUC in the receiver operating characteristic space with $p=0.023$ (Fig. 4). The AUC is equal to the probability that a method will rank a randomly chosen positive instance higher than a randomly chosen negative one [27]. Therefore, the AUC, the surrogate of the accuracy of these two methods, is suitable for comparing their difference.

The improvement of accuracy from the multistage approach ranged from $3 \sim 4 \%$ and $6 \sim 10 \%$ in comparison with single factor cut at $6 \mathrm{~mm}$ and $5 \mathrm{~mm}$, respectively (Tables 2 and 3). This improvement is crucial for specialists dealing with newly diagnosed NPC patients. Reduced sensitivity loss can lower the fear of missing tumor while RT is planning. The involvement of RPLN implies N1 and stage II at least [22]. Chemotherapy is favored to be added to RT due to its association with a higher rate of M1 [15]. A Higher dose of RT to LRPNs could lead to detrimental sequelae such as aspiration, vessel stenosis even stroke, trismus, and cranial cervical nerve injury due to location proximity [28-30]. The miseries from these complications usually are chronic since cancer treatment often leads to long-term survival in NPC patients. A higher RT dose is suggested for positive nodes and vice versa, either over- and under-irradiation can be a serious result. The accurate diagnosis of an LRPN is very important and means that we should jump at any chance to improve it.

The limitations of this study included the lack of radiologic-histopathologic correlation data. The feasibility of histological confirmation before ongoing chemotherapy or RT for LRPNs in newly diagnosed NPC is still lacking, although several biopsy methods had been reported; still, several procedures could be safe [31-35]. However, these procedures are only applied to the recurrent LRPNs and not for new cases before RT. The typical images of our nodes agreed with the positive nodes by RT response follow-up data. Using the criteria for assessing LRPNs with RT response remains the robust standard methodology 
[14]. Second, the cases in our series were limited. Thus, different cohorts must be recruited to determine whether consistent results could be met. We did not have PET/magnetic resonance, which could aid in the evaluation of the mean SUV data in small nodes such as LRPNs [36-38]. We also did not consider other parameters, such as diffusion-weighted imaging in MRI and the effect of Epstein-Barr virus DNA serum titer $[39,40]$. These parameters could be applied in future studies. Finally, the obtained accuracy improvement from adopting the multistage method was relatively small but statistically significant. Given the lack of feasibility of resorting to the final judgment of an LRPN to histological confirmation as a backup in clinical treatment decision making for newly diagnosed NPC patients, clinicians are encouraged to apply this new method despite its long period of operation.

\section{Conclusion}

As a single cutoff value for MIAD, $6 \mathrm{~mm}$ is better than $5 \mathrm{~mm}$. This study supported the advantage of the multistage approach. LRPNs in NPC patients with a MIAD $\geq 6.1 \mathrm{~mm}$ should determine as positive. Among nodes with a MIAD $<6.1 \mathrm{~mm}$, if the mean SUV $\geq 2.6$ or maximal coronal diameter $\geq 25 \mathrm{~mm}$ and maximal axial diameter $\geq 8 \mathrm{~mm}$, the nodes should also be considered as positive.

\section{Supplementary Material}

Supplementary tables.

http://www.medsci.org/v18p3463s1.pdf

Supplementary figures.

http://www.medsci.org/v18p3463s2.xlsx

\section{Acknowledgements}

This work (Yu-Wen Wang) was sponsored by Chi Mei Medical Center, Liouying, Taiwan (grants CLFHR10807). We ensure that no additional external funding was received for this study. These funders had no role in study design, data collection and analysis, decision to publish, or preparation of the manuscript. The authors deeply appreciate the manuscript preparation assistance from Chien-Yu Lin and Chao-Yu Hsu.

\section{Authors' contributions}

DGT, WJY, CHC, and YWW conceived the study concept and initiated the study design. DGT drafted this paper. HYC helped in the analysis and interpretation of data. YSH, YKC, and YWW made contributions to the acquisition and analysis of data. (For MRI and the corresponding FDG PET/CT evaluation, Yu-Wen Wang and Yu-Cheng Hung). YWW was the grant holder. YWW and HYC were involved in study implementation and critically reviewed the manuscript. All authors reviewed the manuscript and gave final approval of the manuscript.

\section{Competing Interests}

The authors have declared that no competing interest exists.

\section{References}

1. Yu E, O'Sullivan B, Kim J, Siu L, Bartlett E. Magnetic resonance imaging of nasopharyngeal carcinoma. Expert Rev Anticancer Ther. 2010; 10: 365-75.

2. Lam WW, Chan YL, Leung SF, Metreweli C. Retropharyngeal lymphadenopathy in nasopharyngeal carcinoma. Head Neck. 1997; 19: 176-81.

3. King AD, Ahuja AT, Leung SF, Lam WW, Teo P, Chan YL, Metreweli C. Neck node metastases from nasopharyngeal carcinoma: MR imaging of patterns of disease. Head Neck. 2000; 22: 275-81.

4. Ng WT, Lee AW, Kan WK, Chan J, Pang ES, Yau TK, et al. N-staging by magnetic resonance imaging for patients with nasopharyngeal carcinoma: pattern of nodal involvement by radiological levels. Radiother Oncol. 2007; 82: $70-5$

5. Coskun HH, Ferlito A, Medina JE, Robbins KT, Rodrigo JP, Strojan P, et al. Retropharyngeal lymph node metastases in head and neck malignancies. Head Neck. 2011; 33: 1520-9.

6. Ou XM, Shen CY, Kong L, Wang XS, Ding JH, Gao YS, et al. Treatment outcome of nasopharyngeal carcinoma with retropharyngeal lymph nodes metastasis only and the feasibility of elective neck irradiation. Oral Oncol. 2012; 48: 1045-50.

7. Hu WX, Zhu GP, Guan XY, Wang XS, Hu CS. The feasibility of omitting irradiation to the contralateral lower neck in stage N1 nasopharyngeal carcinoma patients. Radiat Oncol J. 2013; 8: 230

8. Wu Z, Deng XY, Zeng RF, Su Y, Gu MF, Zhang Y, et al. Analysis of risk factors for retropharyngeal lymph node metastasis in carcinoma of the hypopharynx. Head Neck. 2013; 35: 1274-7.

9. Razek A, Kamal E. Nasopharyngeal carcinoma: correlation of apparent diffusion coefficient value with prognostic parameters. Radiologia Medica. 2013; 118: 534-9.

10. Tang LL, Guo R, Zhou GQ, Sun Y, Liu LZ, Lin AH, et al. Prognostic Value and Staging Classification of Retropharyngeal Lymph Node Metastasis in Nasopharyngeal Carcinoma Patients Treated with Intensity-modulated Radiotherapy. PLoS One. 2014; 9: 108375.

11. Shi Q, Shen CY, Kong L, Wang XS, Ding JH, Gao YS, et al. Involvement of both Cervical Lymph Nodes and Retropharyngeal Lymph Nodes has prognostic value for N1 patients with Nasopharyngeal Carcinoma. Radiat Oncol J. 2014; 9: 7

12. Wu IS, Hung GU, Chang BL, Liu CK, Chang TH, Lee HS, et al. Is unenhanced 18F-FDG-PET/CT better than enhanced CT in the detection of retropharyngeal lymph node metastasis in nasopharyngeal carcinoma? Ear Nose Throat J. 2016; 95: 178-84.

13. Zhang GY, Liu LZ, Wei WH, Deng YM, Li YZ, Liu XW. Radiologic criteria of retropharyngeal lymph node metastasis in nasopharyngeal carcinoma treated with radiation therapy. Radiology. 2010; 255: 605-12.

14. Sharma M, Bartlett E, Yu E. Metastatic retropharyngeal lymph nodes in nasopharyngeal carcinoma: imaging criteria. Expert Rev Anticancer Ther. 2010; 10: 1703-6.

15. Li YZ, Xie CM, Wu YP, Cui CY, Huang ZL, Lu CY, et al. Nasopharyngeal carcinoma patients with retropharyngeal lymph node metastases: a minimum axial diameter of $6 \mathrm{~mm}$ is a more accurate prognostic predictor than $5 \mathrm{~mm}$. AJR Am J Roentgenol. 2015; 204: 20-3.

16. Lee AW, Ng WT, Pan JJ, Poh SS, Ahn YC, AlHussain H, et al. International guideline for the delineation of the clinical target volumes (CTV) for nasopharyngeal carcinoma. Radiother Oncol. 2018; 126: 25-36.

17. Ho FCH, Tham IWK, Earnest A, Lee KM, Lu JDJ. Patterns of regional lymph node metastasis of nasopharyngeal carcinoma: A meta-analysis of clinical evidence. BMC cancer. 2012; 12: 98.

18. Zeng L, Sun XM, Chen CY, Han F, Huang Y, Xiao WW, et al. Comparative study on prophylactic irradiation to the whole neck and to the upper neck for patients with neck lymph node-negative nasopharyngeal carcinoma. Head Neck. 2014; 36: 687-93

19. Wang HZ, Cao CN, Luo JW, Yi JL, Huang XD, Zhang SP, et al. High-risk factors of parotid lymph node metastasis in nasopharyngeal carcinoma: a case-control study. Radiat Oncol J. 2016; 11: 113

20. Bunch PM. Anatomic Eponyms in Neuroradiology: Head and Neck. Acad Radiol. 2016; 23: 1319-32.

21. Huang L, Zhang Y, Liu Y, Li H, Wang S, Liang S, et al. Prognostic value of retropharyngeal lymph node metastasis laterality in nasopharyngeal carcinoma and a proposed modification to the UICC/AJCC N staging system. Radiother Oncol. 2019; 140: 90-7. 
22. Lee AWM, LydiattWM, Colevas D, et al. Nasopharynx. In: Amin MB ES, Greene FL, Byrd DR, et al. ed. AJCC Cancer Staging Manual. 8th ed. Chicago, USA: Springer; 2017: 103-11.

23. Wang YW, Wu CS, Zhang GY, Chang CH, Cheng KS, Yao WI, et al. Can Parameters Other than Minimal Axial Diameter in MRI and PET/CT Further Improve Diagnostic Accuracy for Equivocal Retropharyngeal Lymph Nodes in Nasopharyngeal Carcinoma? PLoS One. 2016; 11: e0163741.

24. Wang YW, Wu CS, Chang CH, Cheng KS, Chang YK, Huang IW, et al. Partial Volume Correction for Equivocal Retropharyngeal Nodal Metastases of Nasopharyngeal Carcinoma with Fluorodeoxyglucose Positron Emission Tomography-Computed Tomography. J Med Biol Eng. 2015; 35: 218-25.

25. Miller $A B$, Hoogstraten $B$, Staquet M, Winkler A. Reporting results of cancer treatment. Cancer. 1981; 47: 207-14.

26. Vellayappan BA, Soon YY, Earnest A, Zhang Q, Koh WY, Tham IWK, et al. Accuracy of (18)F-flurodeoxyglucose-positron emission tomography/ computed tomography in the staging of newly diagnosed nasopharyngeal carcinoma: a systematic review and meta-analysis. Radiol Oncol. 2014; 48: 331-38.

27 Fawcett T. An introduction to ROC analysis. Pattern Recognit Lett. 2006; 27: 861-74.

28. Ng LK, Lee KY, Chiu SN, Ku PK, van Hasselt CA, Tong MC. Silent aspiration and swallowing physiology after radiotherapy in patients with nasopharyngeal carcinoma. Head Neck 2011; 33: 1335-9.

29. Lee CC, Su YC, Ho HC, Hung SK, Lee MS, Chiou WY, et al. Increased risk of ischemic stroke in young nasopharyngeal carcinoma patients. Int J Radiat Oncol Biol Phys. 2011; 81: E833-8.

30. Luk YS, Shum JSF, Sze HCK, Chan LLK, Ng WT, Lee AWM, Predictive factors and radiological features of radiation-induced cranial nerve palsy in patients with nasopharyngeal carcinoma following radical radiotherapy. Oral Oncol. 2013; 49: 49-54.

31. Chan JYW, Chow VLY, Wong STS, Wei WI. Surgical salvage for recurrent retropharyngeal lymph node metastasis in nasopharyngeal carcinoma. Head Neck. 2013; 35: 1726-31.

32. Li JJ, He LJ, Luo GY, Liu LZ, Huang XX, Pan K, et al. Fine-needle aspiration of a retropharyngeal lymph node guided by endoscopic ultrasonography. Endoscopy. 2015; 47: E449-50.

33. Tsai MC, Shu YC, Hsu CC, Lin CK, Lee JC, Chu YH, et al. False-positive finding of retropharyngeal lymph node recurrence in both fluorine (18)FDG PET and MRI in a patient with nasopharyngeal carcinoma. Head Neck. 2016; 38: E84-6.

34. Su Y, Zhao C, Li WJ, Deng XY, Zeng RF, Cui NJ, et al. CT-guided needle biopsy through mandibular area for the diagnosis of nasopharyngeal carcinoma in the parapharyngeal space. Chin J Cancer. 2010; 29: 768-73.

35. He LJ, Xie C, Li Y, Luo LN, Pan K, Gao XY, et al. Ultrasound-guided fine needle aspiration of retropharyngeal lymph nodes after radiotherapy for nasopharyngeal carcinoma: a novel technique for accurate diagnosis. Cancer Commun (Lond). 2018; 38: 20.

36. Becker M, Zaidi H. Imaging in head and neck squamous cell carcinoma: the potential role of PET/MRI. Br J Radiol. 2014; 87: 20130677.

37. Queiroz MA, Huellner MW. PET/MR in Cancers of the Head and Neck. Semin Nucl Med. 2015; 4: 248-65.

38. Fraum TJ, Fowler KJ, McConathy J. PET/MRI: Emerging Clinical Applications in Oncology. Acad Radiol. 2016; 23: 220-36.

39. Varoquaux A, Rager O, Dulguerov P, Burkhardt K, Ailianou A, Becker M. Diffusion-weighted and PET/MR Imaging after Radiation Therapy for Malignant Head and Neck Tumors. Radiographics. 2015; 35: 1502-27.

40. Lin JC, Wang WY, Chen KY, Wei YH, Liang WM, Jan JS, et al. Quantification of plasma Epstein-Barr virus DNA in patients with advanced nasopharyngeal carcinoma. N Engl J Med. 2004; 350: 2461-70. 